\title{
The prevalence of compulsive buying: a meta-analysis
}

\author{
Aniko Maraz ${ }^{1,2}$, Mark D. Griffiths ${ }^{3}$ \& Zsolt Demetrovics' \\ Institute of Psychology, Eötvös Loránd University, Budapest, Hungary, 'Doctoral School of Psychology, Eötvös Loránd University, Budapest, Hungary² and Psychology \\ Division, Nottingham Trent University, Nottingham, UK ${ }^{3}$
}

\section{ABSTRACT}

Aims To estimate the pooled prevalence of compulsive buying behaviour (CBB) in different populations and to determine the effect of age, gender, location and screening instrument on the reported heterogeneity in estimates of CBB and whether publication bias could be identified. Methods Three databases were searched (Medline, PsychInfo, Web of Science) using the terms 'compulsive buying', 'pathological buying' and 'compulsive shopping' to estimate the pooled prevalence of CBB in different populations. Forty studies reporting 49 prevalence estimates from 16 countries were located $(n=32000)$. To conduct the meta-analysis, data from non-clinical studies regarding mean age and gender proportion, geographical study location and screening instrument used to assess CBB were extracted by multiple independent observers and evaluated using a random-effects model. Four a priori subgroups were analysed using pooled estimation (Cohen's $Q$ ) and covariate testing (moderator and meta-regression analysis). Results The CBB pooled prevalence of adult representative studies was 4.9\% (3.4-6.9\%, eight estimates, 10102 participants), although estimates were higher among university students: 8.3\% (5.9-11.5\%, 19 estimates, 14947 participants) in adult non-representative samples: 12.3\% (7.6-19.1\%, 11 estimates, 3929 participants) and in shopping-specific samples: 16.2\% (8.8-27.8\%, 11 estimates, 4686 participants). Being young and female were associated with increased tendency, but not location (United States versus non-United States). Meta-regression revealed large heterogeneity within subgroups, due mainly to diverse measures and time-frames (current versus life-time) used to assess CBB. Conclusions A pooled estimate of compulsive buying behaviour in the populations studied is approximately $5 \%$, but there is large variation between samples accounted for largely by use of different time-frames and measures.

Keywords Addictive behaviour, consumer behaviour, cross-cultural comparison, epidemiology, publication bias, shopping addiction.

Correspondence to: Aniko Maraz, Department of Clinical Psychology and Addiction, Institute of Psychology, Eötvös Loránd University, Izabella u. 46, Budapest, Hungary. E-mail: aniko.maraz@ppk.elte.hu

Submitted 8 June 2015; initial review completed 3 August 2015; final version accepted 27 October 2015

\section{INTRODUCTION}

Research into shopping has demonstrated that although shopping is a necessity in modern life, for some people it is also a leisure activity and a form of entertainment with a rewarding value [1]. However, when taken to the extreme, shopping (or buying) can be a harmful and destructive activity for a minority of individuals. The consequences of compulsive buying behaviour are often underestimated. Christenson et al. [2] noted that CBB results in: (i) large debts $(58.3 \%)$, (ii) inability to meet payments $(41.7 \%)$, (iii) criticism from acquaintances (33.3\%), (iv) legal and financial consequences $(8.3 \%)$, (v) criminal legal problems $(8.3 \%)$ and (vi) guilt (45.8\%). Furthermore, individuals with $\mathrm{CBB}$ often describe an increasing level of urge or anxiety that can be alleviated and lead to a sense of completion only when a purchase is made [3]. Compulsive buying is a frequent disorder in a small minority of shopping mall visitors and is associated with important and robust indicators of psychopathology such as psychiatric distress, borderline personality disorder and substance abuse [4]. Compared to non-compulsive buyers, compulsive buyers are more than twice as likely to abuse substances and have any mood or anxiety disorder and three times more likely to develop eating disorder than non-compulsive buyers [5]. However, it should be noted that these findings are based on a small number of studies, all of which have sampling limitations.

Despite many studies highlighting the severe negative consequences to which compulsive buying can lead, the latest (fifth) edition of the Diagnostic and Statistical Manual of Mental Disorders (DSM-5) does not include 
compulsive buying disorder due to insufficient research in the field [6]. Individuals with the condition are classified within the residual category of 'Unspecified disruptive, impulse-control, and conduct disorders'. Nevertheless, there are various consensus-based definitions of compulsive buying in the research literature. According to Faber, O'Guinn \& Krych [7], compulsive consumption corresponds to a consumer behaviour that is 'inappropriate, typically excessive, and clearly disruptive to the lives of individuals who appear impulsively driven to consume' (p. 132).

McElroy et al. [8] point out that both cognitive and behavioural components play an important role in the acquisition, development and maintenance of the disorder. Diagnostic criteria include: (i) maladaptive preoccupation with buying or shopping or maladaptive buying or shopping impulses; (ii) generation of marked distress by the buying preoccupations, impulses or behaviours, which are time-consuming, interfere significantly with social or occupational functioning or result in financial problems; and (iii) lack of restriction of the excessive buying or shopping behaviour to periods of hypomania or mania. Given the lack of consensus regarding the term, the present study included all pathological consumer behaviour under the umbrella term of 'compulsive buying behaviour' (CBB).

The age of onset for CBB appears to be in the late teens or early 20s, although some studies have reported a later mean age of 30 years [8]. There is also a lack of consensus relating to gender differences. Most clinical studies report that women are much more likely to become compulsive buyers than men, but not all surveys have found significant differences in buying tendencies between men and women [9]. Cultural mechanisms have been proposed to recognize the fact that $\mathrm{CBB}$ occurs mainly among individuals living in developed countries [10]. Elements reported as being necessary for the development of CBB include the presence of a market-based economy, the availability of a wide variety of goods, disposable income and significant leisure time [3]. For these reasons, Black [3] concluded that CBB is unlikely to occur in poorly developed countries, except among the wealthy elite.

Given this background, the main aim of the present paper is to review and summarize the empirical data concerning the prevalence of compulsive buying in non-clinical populations. Following a systematic literature review, the present study (a) estimated a pooled prevalence of $\mathrm{CBB}$ in different populations across the world where studies have been carried out; furthermore, the study examined (b) the effect of age, gender, geographical location of the study and the screening instrument used on the reported heterogeneity in estimates; and (c) whether publication bias could be identified.

\section{METHODS}

\section{Sources and search terms}

This systematic review conforms to the guidelines of metaanalyses in epidemiology outlined by Stroup et al. [11]. At the end of March 2015, three academic databases [Medline (PubMed), PsycInfo and Web of Science] were used to identify all possible papers concerning CBB. The terms applied were 'compulsive buying', 'compulsive shopping' or 'pathological buying'. Searching all fields in the three aforementioned databases resulted in 290, 523 and 449 relevant hits, respectively. Although the aforementioned databases incorporate 'grey literature' such as dissertations and conference presentation, these were excluded later. No further grey literature was searched. After removing duplicates, 638 papers were left for further evaluation.

\section{Inclusion and exclusion criteria}

Inclusion and exclusion criteria were established to maximize specificity and sensitivity across the identified papers. Studies in the papers were considered relevant if they reported empirical prevalence data concerning compulsive buying as well as data from other peer-reviewed works (book chapters, letters to the editor, etc.). The conventional formula was used to calculate the minimum required sample size $[12,13]$ setting precision to $5 \%$, confidence interval (CI) to $99 \%$ and expected prevalence to $5.8 \%$, that are the most recent representative prevalence data for the United States. Using the given formula, studies with 145 or more participants were considered suitable to return reliable prevalence rates. For this reason, in order to be included in the current review, studies had to be carried out on a non-clinical sample with more than 145 participants. Only published studies written in English were included, with no constraints regarding participants' ages. Where multiple publications presented identical data, the most 'informative version' of the study was included so as not to doublecount what was, in reality, a single prevalence estimate. Two authors categorized the search results (A.M. and Z.D.) and a third author was included in cases of disagreement (M.D.G.).

As noted in the previous section, 638 publications were identified. The first papers excluded were case studies $(n=23)$ and reviews or theoretical works $(n=192)$ that included no new empirical data. In the next step, a further 244 clinical samples were excluded, such as studies examining drug effectiveness and comparing compulsive buyers with other populations, as well as those with other mental health issues such as Parkinson's disease or eating disorders. A further 26 studies were excluded because they disseminated qualitative findings or double-reported prevalence data from the same sample. Another 73 papers were excluded because they used the compulsive buying scale 
points as a measure of severity, and did not report the prevalence rates. The original 638 publications also included 15 written in a foreign language, seven dissertation or conference abstracts and 16 studies in which the sample size was below 145 participants. Given that the present review focuses on adult populations, two studies that recruited high school children were also excluded (i.e. $[14,15]){ }^{1}$ This left 40 studies that met the predetermined inclusion criteria for the current review.

\section{Meta-analysis: data analysis}

The following information was extracted from the studies: sample mean age, proportion of females (in \%), the study's geographical location and the screening instrument used to assess CBB, and the reported prevalence estimate of CBB. Furthermore, the association between age, gender and CBB was also extracted as reported by the individual studies.

The unit of data analysis was the estimated prevalence rate for $\mathrm{CBB}$ and not the studies. This was because some studies reported more than one prevalence rate for the same sample. This approach was opted for in order to avoid bias as a result of having to choose between the estimates assessed by the different CBB screening measures. Given that inherently different populations are clearly not comparable, the following subgroups were formed a priori: adult representative samples, adult non-representative samples (e.g. university staff members), university student samples and shopping-specific samples (e.g. customers of a shopping mall). Using the random-effects model, studies were weighted by the inverse of their variance, so that studies with larger sample sizes and more accurate estimates of population parameters had a greater weight on the mean effect size. Prevalence estimates were considered outliers if the standardized residual exceeded \pm 3.29 [16]. In the current study, no outliers were identified and all standardized residuals were within the acceptable range.

To address the issue of publication bias, a funnel plot asymmetry was examined following the guidelines by Sterne et al. [17]. A funnel plot is a scatter-plot of the effect estimates from individual studies against measures of each study's size. In the absence of publication bias and between-study heterogeneity, the scatter will be due to sampling variation alone and the plot will resemble a symmetrical inverted funnel. Following the recent recommendations by Sterne et al. [17], Egger's test of the intercept was used to evaluate publication bias statistically. The more the intercept deviates from zero, the more pronounced the asymmetry. If the $P$-value of the intercept is 0.1 or smaller, the asymmetry is considered to be statistically significant. However, Egger's test, similar to other statistical tests for funnel plot asymmetry, has low statistical power [17].
The rate of heterogeneity was calculated separately within each of the four groups. Heterogeneity refers to the differences between the studies in terms of methods, participants and other unknown sources [18]. This can be tested using the $Q$-statistic with a random-effects model (Cochran's $Q$, see: [19-22]). Cochran's $Q$ has an approximate $\chi^{2}$ distribution and represents the degree of departure from homogeneity. A significant $(P<0.05) Q$-value indicates that there is statistically significant heterogeneity in the studies.

Covariates were tested when heterogeneity was identified within a subgroup. Meta-regression was used to assess the association between outcome (prevalence) and continuous covariates such as sample mean age and the proportion of females [23]. Moderator analysis was used for categorical variables such as study location (i.e. United States versus non-United States) and assessment screening tool used (current versus life-time prevalence). Only moderator variables that had at least four estimates in one cell were used [24]. Moderators were significant in cases of categorical variables if $Q_{\text {between }}$ was significant. The regression coefficient (and its significance level) was calculated in addition to $\mathrm{Tau}^{2}$ and reflects between-study variance. The Comprehensive MetaAnalysis version 3 software $[25,26]$ was used to calculate prevalence estimates within groups and publication bias and to conduct moderator and meta-regression analysis.

\section{RESULTS}

\section{Prevalence by populations}

As noted above, 40 relevant studies were identified that met the inclusion criteria, reporting 49 different prevalence rate estimates for 32333 participants. Table 1 depicts the studies in greater detail. As already noted, the sample was divided a priori into four subsamples: adult representative, adult nonrepresentative, university student and shopping-specific. The mean prevalence of compulsive buying was $4.9 \%$ in adult representative samples $(\mathrm{CI}=3.4-6.9 \%, 10102$ participants), $12.3 \%$ in adult non-representative samples (CI $=7.6-19.1 \%, 3929$ participants), 8.3\% in university student samples (CI: 5.9-11.5\%, 14947 participants) and $16.2 \%$ in shopping-specific samples $(\mathrm{CI}=8.8-27.8 \%$, 4686 participants) (see Fig. 1).

There was significant heterogeneity in each of the four groups (representative: $Q_{\text {within }}=101.4 P<0.001$; non-representative: $Q_{\text {within }}=322.3 P<0.001$; student: $Q_{\text {within }}=604.1 \quad P<0.001 ; \quad$ specific: $\quad Q_{\text {within }}=1038.7$ $P<0.001)$. Thus, covariates were tested to explain variability.

${ }^{1}$ Prevalence rates were: 19\% (Chinese junior high school students), 25\% (Thai junior high school students) [14] and 40\% among Italian upper intermediate school students [15]. 
$4 \quad$ Aniko Maraz et al.

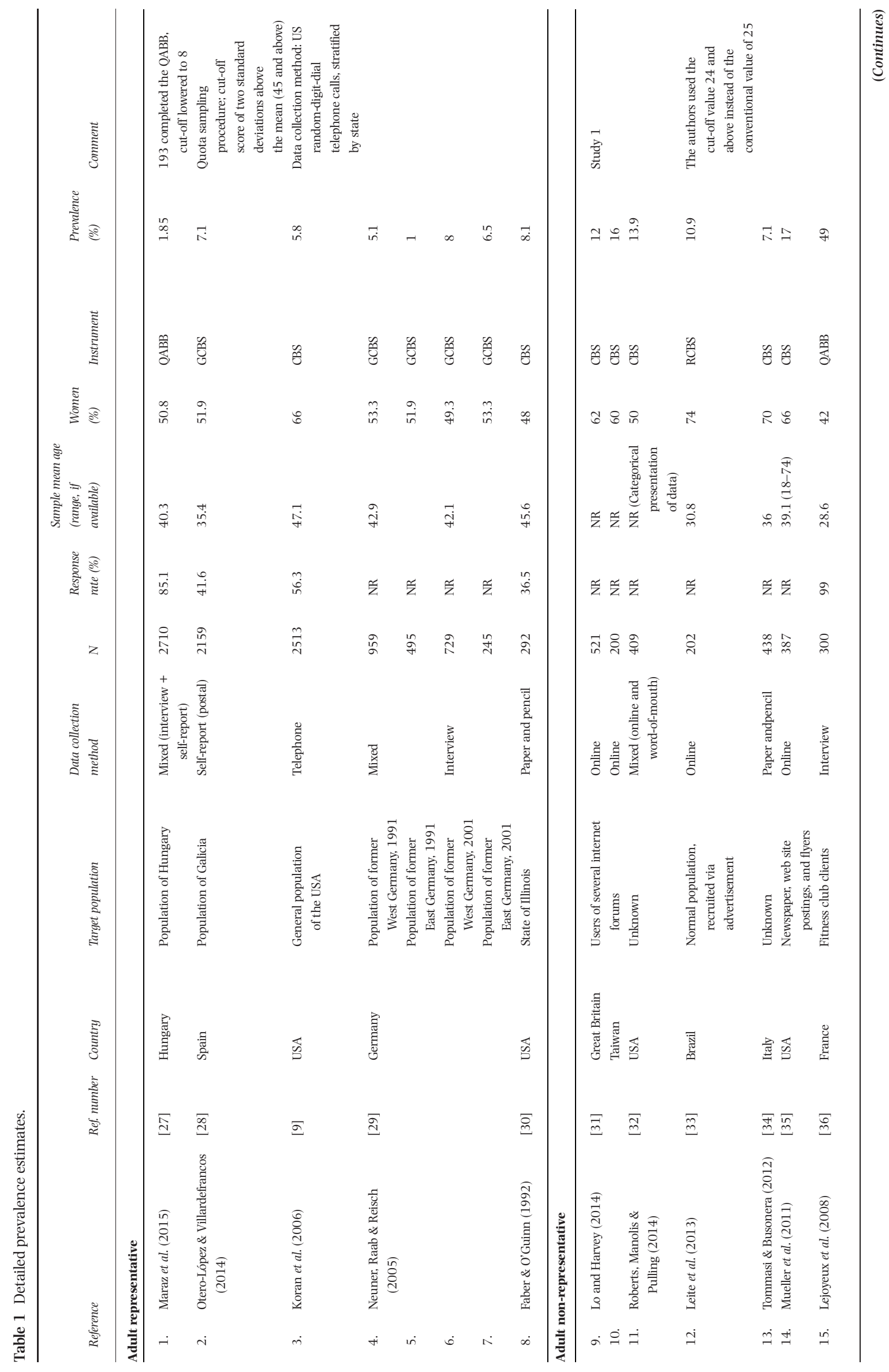




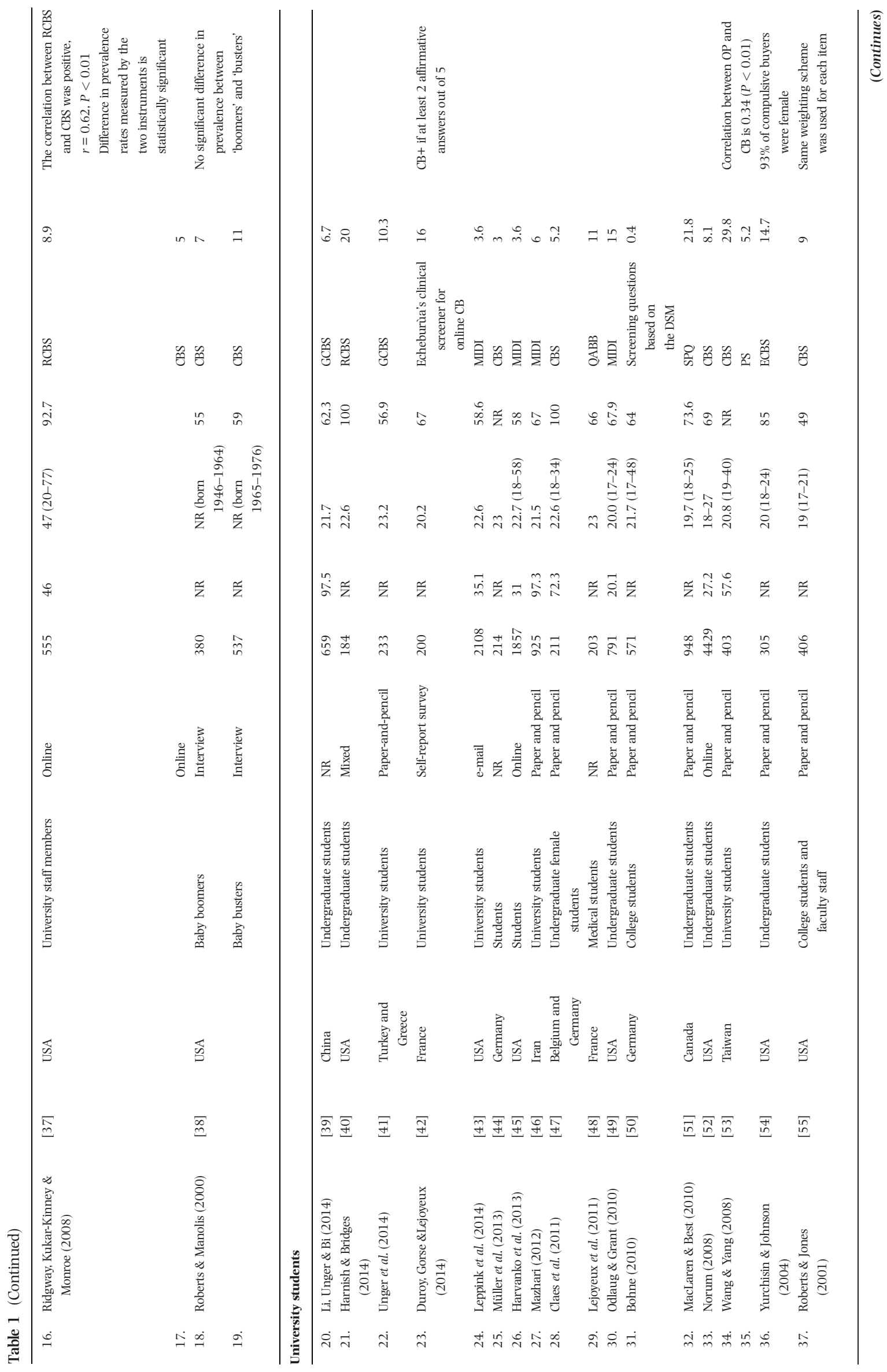




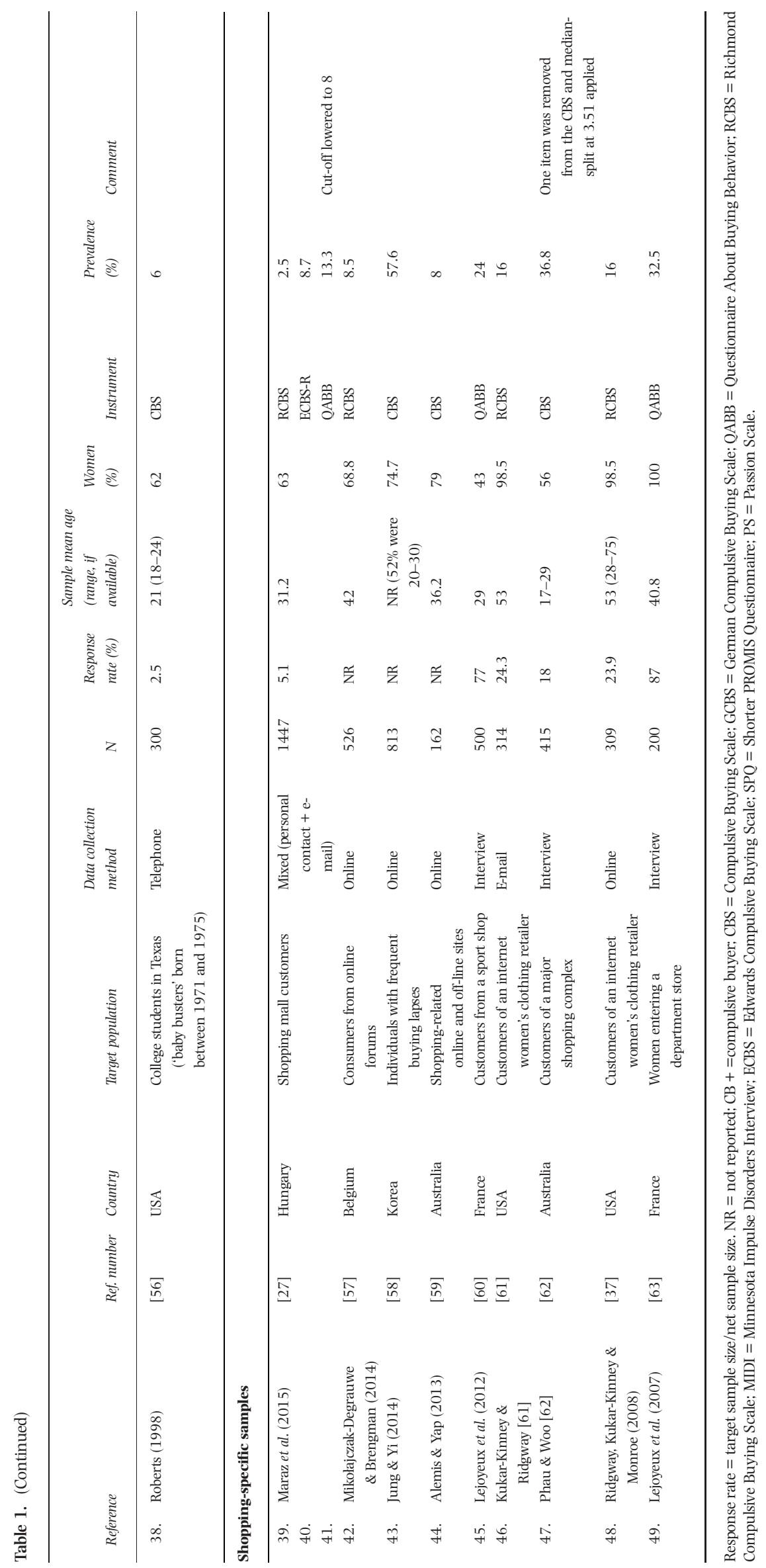




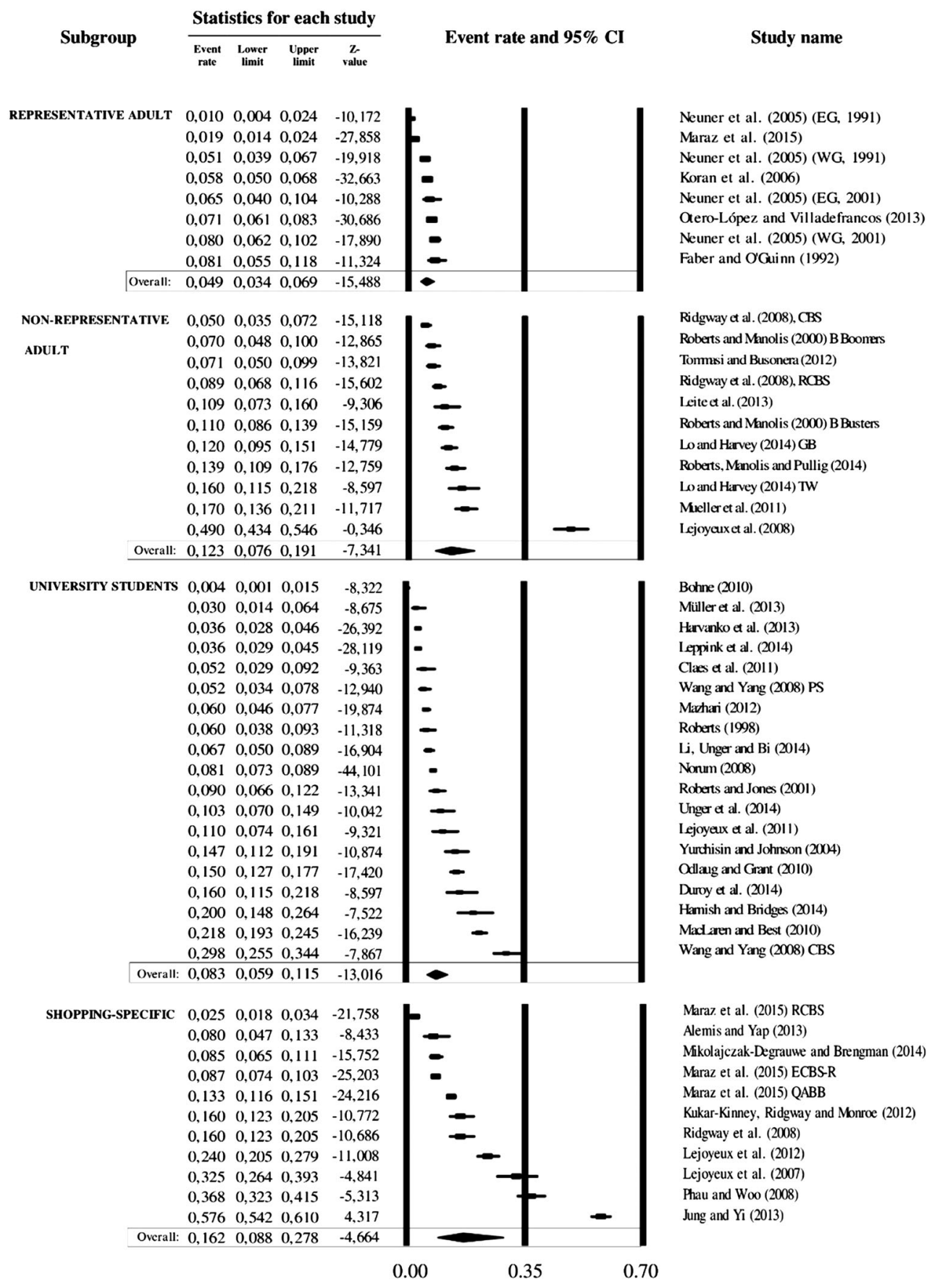

Figure I Forest plot of prevalence estimates in non-clinical populations by subgroup

Publication bias

The funnel plot of standard error was symmetrical in three subgroups (see Supporting information). Following visual inspection, the most symmetrical was the adult representative samples' funnel plot, and the least symmetrical was the plot of the specific samples. Egger's $P$-value indicated significant symmetry in special populations (intercept $=-13.20$, $P=0.15$ ), adult representative (intercept $=-2.96, P=0.39$ ) and student populations (intercept $=-2.47, P=0.38$ ). There was significant asymmetry in the adult non-representative (intercept $=-17.80, P=0.03$ ) and likely to be caused by an extremely high estimate of $49 \%$ in one particular study [36].

Covariate analysis

Age, gender and study location (USA versus non-USA)

In total, in eight different samples, seven studies reported that compulsive buying tendency decreases with age, of which five estimates were reported in adult representative samples. 
Table 2 Assessment tools used to assess compulsive buying and the frequency of usage.

\begin{tabular}{lllr}
\hline & & & Number of \\
Assessment tool & Cut-off & Type of prevalence & prevalence estimates \\
\hline CBS & Clinically valid & Life-time & 19 \\
GCBS & Clinically valid & Current & 7 \\
QABB & Conventional [63] and based on psychometrics [27] & Mixed & 6 \\
RCBS & Clinically valid & Current & 7 \\
MIDI & Based on theory & Life-time & 4 \\
ECBS & Conventional & Current & 1 \\
ECBS-R & Based on psychometrics & Current & 1 \\
SPQ & Based on psychometrics & Life-time & 1 \\
PS & Based on psychometrics & Current & 1 \\
Screening questions based & Based on theory & NR & 1 \\
$\quad$ on the DSM & & & 1 \\
ESOCB & Conventional & Unknown & 49 \\
Total & & & \\
\hline
\end{tabular}

CBS = Compulsive Buying Scale; GCBS = German Compulsive Buying Scale; $\mathrm{QABB}=$ Questionnaire About Buying Behavior; $\mathrm{RCBS}=$ Richmond Compulsive Buying Scale; MIDI = Minnesota Impulse Disorders Interview; ECBS = Edwards Compulsive Shopping Scale; SPQ = Shorter PROMIS Questionnaire, Passion Scale; ESOCB = Echeburua's screener for online CBB; NR = not reported.

Age did not have an effect on CBB in five samples. Only one study reported that older students were more likely to engage in compulsive buying than younger ones in a sample of undergraduate students [52]. The remaining studies did not test or report the association between age and CBB. The mean sample age was lower for adult non-representative and shopping-specific populations than for adult representative ones (weighted means, respectively: 37.4, 37.2 and 41.7 years). Age had non-significant effects in all four populations; in the representative (coefficient: 0.02, $P=0.77$, $Q=0.09, \quad \mathrm{Tau}^{2}=0.38$ ), non-representative (coefficient: -0.08, $P=0.21$, $\mathrm{Tau}^{2}=0.85$ ), student (coefficient: $-0.24, P=0.051$, Tau $\left.{ }^{2}=0.36\right)$ and shopping-specific samples (coefficient: 0.03, $P=0.38$, $\mathrm{Tau}^{2}=0.58$ ).

With regard to gender, women were more prone to $\mathrm{CBB}$ than men in 12 different samples, four of which were reported on adult representative samples. No gender difference was found in four instances, and in one sample undergraduate men reported higher CBB tendencies than women [52]. On average, the samples included more females, including $55.5 \%$ of adult representative, $69.4 \%$ of adult non-representative, $65.9 \%$ of university student and $69.8 \%$ of shopping-specific samples. The proportion of females in the sample had non-significant effects in the representative (coefficient: $-0.002, P=0.96$, $\mathrm{Tau}^{2}=0.35$ ), student (coefficient: 0.02, $P=0.10$, $\mathrm{Tau}^{2}=0.45$ ) and specific populations (coefficient: $0.013, P=0.33$, $\mathrm{Tau}^{2}=0.58$ ), but significant effect in the non-representative subgroup (coefficient $=-0.05, P<0.01, \mathrm{Tau}^{2}=0.32$ ). The higher the proportion of females, the lower the reported prevalence of compulsive buying in the adult nonrepresentative sample.

With regard to the geographic location of where the study was carried out, most estimates $(n=18)$ were reported from the United States, followed by Germany $(n=6)$, France $(n=5)$ and Hungary $(n=4)$. Tested as moderators, United States versus non-United States study location was calculated $(n \geq 4)$, the effect of which was non-significant in two groups: in the non-representative (point estimates: 0.10 and 0.16, $Q_{\text {between }}=0.957$, $P=0.33$ ) and in the student population (point estimates: 0.09 and $\left.0.08, Q_{\text {between }}=0.05, P=0.82\right){ }^{2}$

The effect of assessment tool: life-time versus current prevalence

As shown in Table 2, 39\% of the prevalence rate estimates (19 of 49) were obtained using the CBS [30], although cutoff scores differed. A considerable amount of variability in estimated prevalence rates was due most probably to the fact that measures had different time-frames. The CBS, MIDI and Shorter PROMIS Questionnaire (SPQ) contain items regarding life-time $\mathrm{CBB}$ prevalence (that is, if the individual has ever experienced problems with buying behaviour), whereas the German Compulsive Buying Scale (G-CBS), Richmond Compulsive Buying Scale (RCBS), Edwards Compulsive Buying Scale (ECBS), ECBS-R and the Passion Scale (PS) assess current CBB prevalence (problems with buying behaviour at the time of assessment). The QABB contains mixed items regarding verb

\footnotetext{
${ }^{2}$ In the representative populations, estimates were 0.07 and 0.04 but $n=2$ and $n=6$, respectively, for United States versus non-United States, whereas in the specific populations point estimates were both 0.16 , but $n=2$ and $n=9$.
} 
tense (i.e. 13 of the 19 items refer to past, and six refer to present behaviour). When calculating the mean average estimates by the type of instrument, the sample sizeweighted mean of current estimate was $6.99 \%$ (assessed by GCBS, RCBS, ECBS, ECBS-R or PS), life-time estimate was $11.08 \%$ (assessed by CBS, MIDI or SPQ) and the mixed estimate was 11.14\% [assessed by Questionnaire About Buying Behavior (QABB)].

Unfortunately, there were fewer than four studies in three of the four groups, therefore differences between estimates (life-time versus current) could be calculated in only one subgroup. Among students, point estimates were 0.09 and 0.08 for current and life-time prevalence, respectively, which yielded a non-significant difference $(Q=0.73$, $P=0.70) .{ }^{3}$ However, as a trend, life-time prevalence estimates were clearly higher than current estimates.

\section{DISCUSSION}

The present review aimed to summarize knowledge concerning the prevalence of compulsive buying in nonclinical adult populations. It also aimed to examine the possible causes of the varying estimates of CBB disorder and to calculate a pooled prevalence based on all existing prevalence data. Via systematic literature review, 40 relevant studies were identified reporting 49 different estimates for more than 32000 participants. The estimated prevalence rate of compulsive buying was $4.9 \%$ (3.4-6.9\%, based on eight estimates) in the general adult representative populations. Prevalence rates were higher in university student samples [8.3\% (5.9-11.5\%), 4686 estimates] and in adult non-representative samples [12.3\% (7.6-19.1\%) 11 estimates] compared to representative ones. Unsurprisingly, the highest prevalence rates were among shoppingspecific samples [(16.2\% (8.8-27.8\%), 11 estimates].

A considerable amount of heterogeneity was present in the reported estimates and was also reflected by the funnel plots. Funnel plots indicated significant asymmetry only in the adult non-representative samples, due mainly to one (although statistically speaking non-outlier) extremely high value of $49 \%$ among fitness club clients [36]. Although asymmetrical funnel plots are interpreted as indicators of publication bias, they may give the wrong impression if high precision studies are different from low precision studies with respect to effect size (e.g. due to different populations examined) [64]. Therefore, in this case heterogeneity is likely to be accounted for by the heterogeneous populations and instruments rather than by publication bias.
On one hand, heterogeneity in prevalence rates may be accounted for by the lack of consensus regarding the definition of compulsive buying. Studies used different measures to assess CBB, each having a different conceptual background. Most definitions include cognitive-affective indicators as well as maladaptive behavioural consequences when defining the disorder (e.g. debts). The screening instruments used across studies differed in indicators of financial consequences (e.g. credit card use, debts, loan, etc.) and are subject to differences according to countries, subcultures and/or age groups. Given the challenges of establishing a reliable cut-off value for the scales, about twice as many studies $(n=73)$ used the rating scales as indicators of severity (ignoring the cut-off values) as opposed to those that reported categorical data $(n=40)$. Researchers have noted repeatedly that compulsive buying tendencies vary along a spectrum $[65,66]$, and they argue that psychological problems exist as dimensions rather than categories. However, a categorical approach has the advantage of identifying potentially self-harming individuals as well as an estimation of the problem extent within the given population. Furthermore, knowledge of the proportion of compulsive buyers in the study sample enables comparison across studies. Therefore, future studies should report the proportion of compulsive buyers in their samples.

On the other hand, cut-off scores were not standard, but differed across measures. In relation to the Compulsive Buying Scale, Faber \& O'Guinn [30] noted that 'a cut-off point at 2 SD is recommended for research purposes. This point is associated with a scale score of -1.34 ' (p. 464), thereby suggesting a cut-off value adjusted to the mean score and standard deviation of the given population. The adjustable cut-off score is suitable to account for cultural differences in the indicators of CBB (e.g. the use of credit cards), although this approach was adhered to in only a minority of studies. Application of a fixed cut-off value $(-1.34$ for the CBS) was more common, which sets the same standard of buying pathology across countries without taking into consideration the instrument's local validity and reliability. The reporting of local validity is especially important given the culture-dependent nature of compulsive buying behaviour. For example, some countries have an extended tradition of bank check usage and others do not have. This difference is ignored when administering an instrument where using a bank check is an indicator of CBB (such as the CBS).

Thirdly, diversity is due to the fact that measures do not explicitly distinguish current and life-time assessment of CBB. Prevalence rates assessed with an instrument that

\footnotetext{
${ }^{3}$ Point estimates for the representative group were $0.06(n=5), 0.07(n=2)$, for the non-representative group were $0.10(n=2)$ and $0.11(n=8)$, and for the specific group: $0.09(n=5)$ and $0.30(n=3)$ for current and life-time estimates, respectively. Data obtained via mixed instruments $(Q A B B)$ were treated as missing.
} 
assesses life-time prevalence report 1.6 times higher rates on average than those assessing current prevalence. This proportion is in line with other studies reporting 50\% lifetime and $30 \%$ of 12 -month prevalence of any psychiatric disorder in the United States [67] and 6.9\% life-time versus $3.4 \%$ 12-month prevalence of major depression among Chinese Americans [68]. Pooled life-time versus current estimates in adult representative samples in the present review were 6.1 and $6.0 \%$, respectively (excluding estimates assessed by the QABB), but these estimates were largely varying, therefore more data are needed to establish reliable estimates. Future studies should therefore separate out current and life-time prevalence of the disorder explicitly when assessing CBB.

Non-representative samples (e.g. adults, university students, shoppers) tended to recruit younger participants who were more likely to be female than representative studies. The mean age of the sample and the proportion of males and females did not have a reliable effect on the prevalence estimates. Being of a younger age was predictive of $\mathrm{CBB}$, according to individual study results, and also according to the regression analysis in the representative samples. In the other groups, it is possible that methodological heterogeneity masked the effects of age. However, it remains open as to whether compulsive buying tendency decreases with age or this difference reflects generational differences. If the latter was the case, then the prevalence of compulsive buying behaviour is expected to increase in the future. There is already evidence in the literature for increasing rates of CBB in Germany [29] and in Spain [69], but longitudinal studies are needed to clarify this question. Nevertheless, the tendency of a younger age being associated with higher $\mathrm{CBB}$ tendency is also reflected by the fact that university samples report higher CBB tendencies than do general adult samples. The overwhelming majority of individual studies report that women are more likely to be compulsive buyers than men, although this effect was either non-significant or very weak when tested as a covariate. Again, this is due probably to the large methodological differences in the studies. The dominance of women in $\mathrm{CBB}$ is in line with the evolutionary explanations of the disorder, that $\mathrm{CBB}$ might reflect ancient collecting tendencies that had been assigned mainly to females within their social groupings [70]. In any case, the fact that adult non-representative studies recruit young and female participants is a significant contributory factor to the elevated prevalence rates reported.

In relation to data collection, estimates from the United States (18 of 49) were over-represented compared to countries other than the United States, although there was no difference in the reported estimates between the US and non-US countries. However, it is difficult to draw reliable conclusions regarding the cultural variance of $\mathrm{CBB}$, given that adult representative estimates are only available from the United States, Spain, Germany and Hungary. Future studies are needed to clarify whether the geographical location (or culture) has any effect on the prevalence rates.

Finally, some estimates deviate largely from the expected values within the given subgroup. For example, Lejoyeux et al. [36] reported that $49 \%$ of fitness club clients and 33\% of women entering a department store [63] had CBB. Although these estimates are not representative of the given population, the high estimates raise the concern of overpathologizing the behaviour [71], especially when no other measures (such as overspending) were assessed to validate the category of compulsive buyers.

The present study is not without limitations. The relationship described by a meta-regression is an observational association across trials; therefore, it is unsuitable for causal interpretation. Furthermore, because metaanalyses rely on published data, the results suffer from the same sampling errors and biases in the original observations (aggregation bias, see [22]) Furthermore, not every study was designed to report prevalence, and only the representative estimates are suitable to draw reliable estimates regarding the prevalence rates of $\mathrm{CBB}$. Finally, even the CBS that has good sensitivity (89.8\%) and specificity (85.3\%) values has a positive predictive value of only approximately $20 \%$ when the prevalence is $5 \%$. This means that only one of five individuals who screen positive on CBS is problematic in a clinical sense [72].

The fact that compulsive buying behaviour is a relatively common disorder with severe consequences for a minority of individuals should not be overlooked. It appears that approximately one in 20 individuals suffer from $\mathrm{CBB}$ at some point in their lives, and that being young and female are associated with a higher risk of CBB. High heterogeneity is likely to be the result of methodological variability within studies, such as assessment screens with different time-frames and conceptual background. Future studies should therefore think carefully about how to conceptualize the disorder and to separate out current versus life-time prevalence clearly in the samples used.

\section{Declaration of interests}

None.

\section{Acknowledgements}

The authors would like to thank Dr Zsofia K. Takacs (Eötvös Loránd University, Hungary) for her help with the meta-analysis.

\section{References}

1. Mukhopadhyay A., Johar G. V. Indulgence as self-reward for prior shopping restraint: a justification-based mechanism. J Consult Psychol 2009; 19: 334-45. 
2. Christenson G., Faber R., de Zwaan M., Raymond N. C., Specker S. M., Ekern M. D. et al. Compulsive buying: descriptive characteristics and psychiatric comorbidity. J Clin Psychiatry 1994; 55: 5-11.

3. Black D. W. A review of compulsive buying disorder. World Psychiatry 2007; 6: 14.

4. Maraz A, van den Brink W, Demetrovics Z. Prevalence and construct validity of compulsive buying disorder in shopping mall visitors. Psychiatry Res 2015; 3: 918-24.

5. Black D. W., Repertinger S., Gaffney G. R., Gabel J. Family history and psychiatric comorbidity in persons with compulsive buying: preliminary findings. Am J Psychiatry 1998; 155: 960-3.

6. American Psychiatric Association. Diagnostic and statistical manual of mental disorders (DSM-5), 5th edn. Arlington, VA: American Psychiatric Association; 2013.

7. Faber RJ, O'Guinn TC, Krych R. Compulsive consumption. Adv Consum Res 1987;14:132-5.

8. McElroy S. L., Keck J. P. E., Pope J. H. G., Smith J. M., Strakowski S. M. Compulsive buying: a report of 20 cases. J Clin Psychiatry 1994; 55: 242-8.

9. Koran L. M., Faber R. J., Aboujaoude E., Large M. D., Serpe R. T. Estimated prevalence of compulsive buying behavior in the United States. Am J Psychiatry 2006; 163: 1086-12.

10. Black D. W., Monahan P., Schlosser S., Repertinger S. Compulsive buying severity: an analysis of compulsive buying scale results in 44 subjects. J Nerv Ment Dis 2001; 189: 123-6.

11. Stroup D. F., Berlin J. A., Morton S. C., Olkin I., Williamson G. D., Rennie D. et al. Meta-analysis of observational studies in epidemiology: a proposal for reporting. JAMA 2000; 283: 2008-12.

12. Lwanga SK, Lemeshow S. Sample Size Determination in Health Studies: A Practical Manual. Geneva: World Health Organization; 1991.

13. Naing L., Winn T., Rusli B. Practical issues in calculating the sample size for prevalence studies. Arch Orofacial Sci 2006; 1: 9-14.

14. Guo Z., Cai Y. Exploring the antecedents of compulsive buying tendency among adolescents in China and Thailand: a consumer socialization perspective. Afr J Bus Manage 2011; 5: 10198-209.

15. Villella C., Martinotti G., Di Nicola M., Cassano M., La Torre G., Gliubizzi M. D. et al. Behavioural addictions in adolescents and young adults: results from a prevalence study. J Gambl Stud 2011; 27: 203-14.

16. Tabachnick BG, Fidell AS. Using Multivariate Statistics. Boston, MA: Pearsonn; 2007.

17. Sterne J. A., Sutton A. J., Ioannidis J., Terrin N., Jones D. R., Lau J. et al. Recommendations for examining and interpreting funnel plot asymmetry in meta-analyses of randomised controlled trials. BMJ 2011; 343.

18. Petticrew M, Roberts H. Systematic Reviews in the Social Sciences: a Practical Guide. Chichester: John Wiley \& Sons; 2008.

19. Smith T. C., Spiegelhalter D. J., Thomas A. Bayesian approaches to random-effects meta-analysis: a comparative study. Stat Med 1995; 14: 2685-99.

20. Sutton A. J., Abrams K. R., Jones D. R., Sheldon T. A., Song F. Systematic Reviews of Trials and Other Studies. United Kingdom: Health Technology Assessment; 1998.

21. Higgins J. P. T., Thompson S. G., Deeks J. J., Altman D. G. Measuring inconsistency in meta-analyses. BMJ 2003; 327: 557-60.

22. Higgins JPT. Assessing statistical heterogeneity: chi-squared or I-squared. Newsletter of the Cochrane Wounds Group 2004; 3-4.
23. Thompson S. G., Higgins J. How should meta-regression analyses be undertaken and interpreted? Stat Med 2002; 21: 1559-73.

24. Bakermans-Kranenburg M. J., van Ijzendoorn M. H., Juffer F. Less is more: meta-analyses of sensitivity and attachment interventions in early childhood. Psychol Bull 2003; 129: 195-215.

25. Borenstein M, Hedges L, Higgins J, Rothstein H. Comprehensive Meta-Analysis Version 3. Englewood, NJ: Biostat; 2014.

26. Borenstein M, Hedges L, Higgins J, Rothstein H. Manual to the Comprehensive Meta-Analysis Version 3 software. Englewood, NJ: Biostat; 2015.

27. Maraz A., Eisinger A., Hende B., Urbán R., Paksi B., Kun B. et al. Measuring compulsive buying behaviour: psychometric validity of three different scales and prevalence in the general population and in shopping centres. Psychiatry Res 2015; 225: 326-34.

28. Otero-López J. M., Villardefrancos E. Prevalence, sociodemographic factors, psychological distress, and coping strategies related to compulsive buying: a cross sectional study in Galicia, Spain. BMC Psychiatry 2014; 14: 101.

29. Neuner M., Raab G., Reisch L. A. Compulsive buying in maturing consumer societies: an empirical re-inquiry. $J$ Econ Psychol 2005; 26: 509-22.

30. Faber R. J., O'Guinn T. C. A clinical screener for compulsive buying. J Consum Res 1992; 19: 459-69.

31. Lo H.-Y., Harvey N. Compulsive buying: obsessive acquisition, collecting or hoarding? In J Mental Health Addict 2014; 12: 453-69.

32. Roberts J. A., Manolis C., Pullig C. Contingent self-esteem, self-presentational concerns, and compulsive buying. Psychol Market 2014; 31: 147-60.

33. Leite P., Rangé B., Kukar-Kiney M., Ridgway N., Monroe K., Ribas Junior R. et al. Cross-cultural adaptation, validation and reliability of the Brazilian version of the Richmond compulsive buying scale. Rev Bras Psiquiatr 2013; 35: $38-43$.

34. Tommasi M., Busonera A. Validation of three compulsive buying scales on an Italian sample. Psychol Rep 2012; 111: 831-44.

35. Mueller A., Mitchell J. E., Peterson L. A., Faber R. J., Steffen K. J., Crosby R. D. et al. Depression, materialism, and excessive internet use in relation to compulsive buying. Compr Psychiatry 2011; 52: 420-4.

36. Lejoyeux M., Avril M., Richoux C., Embouazza H., Nivoli F. Prevalence of exercise dependence and other behavioral addictions among clients of a Parisian fitness room. Compr Psychiatry 2008; 49: 353-8.

37. Ridgway N. M., Kukar-Kinney M., Monroe K. B. An expanded conceptualization and a new measure of compulsive buying. J Consum Res 2008; 35: 622-39.

38. Roberts J. A., Manolis C. Baby boomers and busters: an exploratory investigation of attitudes toward marketing, advertising and consumerism. J Consum Market 2000; 17: 481-97.

39. Li S., Unger A., Bi C. Different facets of compulsive buying among Chinese students. J Behav Addict 2014; 3: 238-45.

40. Harnish R. J., Bridges K. R. Compulsive buying: the role of irrational beliefs, materialism, and narcissism. J Ration Emot Cogn Behav Ther 2014; 33: 1-16.

41. Unger A., Papastamatelou J., Okan E. Y., Aytas S. How the economic situation moderates the influence of available money on compulsive buying of students - a comparative study between Turkey and Greece. J Behav Addict 2014; 3: 173-81. 
42. Duroy D., Gorse P., Lejoyeux M. Characteristics of online compulsive buying in Parisian students. Addict Behav 2014; 39: 1827-30.

43. Leppink E., Odlaug B. L., Lust K., Christenson G., Derbyshire K., Grant J. E. Losing control: assaultive behavior as a predictor of impulse control disorders in young adults. Compr Psychiatry 2014; 55: 1831-6.

44. Müller A., Smits D. J., Claes L., Gefeller O., Hinz A., de Zwaan M. The German version of the material values scale. GMS Psychosoc Med 2013; 10: 1-9.

45. Harvanko A., Lust K., Odlaug B. L., Schreiber L. R., Derbyshire K., Christenson G. et al. Prevalence and characteristics of compulsive buying in college students. Psychiatry Res 2013; 210: 1079-85.

46. Mazhari S. Association between problematic internet use and impulse control disorders among Iranian university students. Cyberpsychol Behav Soc Netw 2012; 15: 270-3.

47. Claes L., Bijttebier P., Mitchell J. E., de Zwaan M., Mueller A. The relationship between compulsive buying, eating disorder symptoms, and temperament in a sample of female students. Compr Psychiatry 2011; 52: 50-5.

48. Lejoyeux M., Richoux-Benhaim C., Betizeau A., Lequen V., Lohnhardt H. Money attitude, self-esteem, and compulsive buying in a population of medical students. Front Psychiatry 2011; 2: 13-6.

49. Odlaug B. L., Grant J. E. Impulse-control disorders in a college sample: results from the self-administered Minnesota impulse disorders interview (MIDI). Prim Care Companion J Clin Psychiatry 2010; 12: :PCC.09m00842.

50. Bohne A. Impulse-control disorders in college students. Psychiatry Res 2010; 176: 91-2.

51. MacLaren V. V., Best L. A. Multiple addictive behaviors in young adults: student norms for the shorter PROMIS questionnaire. Addict Behav 2010; 35: 252-5.

52. Norum P. S. Student internet purchases. Fam Consum Sci Res J 2008; 36: 373-88.

53. Wang C.-C., Yang H.-W. Passion for online shopping: the influence of personality and compulsive buying. Soc Behav Pers 2008; 36: 693-706.

54. Yurchisin J., Johnson K. K. Compulsive buying behavior and its relationship to perceived social status associated with buying, materialism, self-esteem, and apparelproduct involvement. Fam Consum Sci Res J 2004; 32: 291-314.

55. Roberts J. A., Jones E. Money attitudes, credit card use, and compulsive buying among American college students. J Consum Aff 2001; 35: 213-40.

56. Roberts J. A. Compulsive buying among college students: an investigation of its antedecents, consequences, and implications for public policy. J Consum Aff 1998; 32: 295-319.

57. Mikołajczak-Degrauwe K., Brengman M. The influence of advertising on compulsive buying - the role of persuasion knowledge. J Behav Addict 2014; 3: 65-73.

58. Jung J., Yi S. Assessment of heterogeneity of compulsive buyers based on affective antecedents of buying lapses. Addiction Res Theor 2014; 22: 37-48.
59. Alemis M. C., Yap K. The role of negative urgency impulsivity and financial management practices in compulsive buying. Aust J Psychol 2013; 65: 224-31.

60. Lejoyeux M., Guillot C., Chalvin F., Petit A., Lequen V. Exercise dependence among customers from a Parisian sport shop. J Behav Addict 2012; 1: 28-34.

61. Kukar-Kinney M., Ridgway N. M., Monroe K. B. The role of price in the behavior and purchase decisions of compulsive buyers. J Retailing 2012; 88: 63-71.

62. Phau I., Woo C. Understanding compulsive buying tendencies among young Australians: the roles of money attitude and credit card usage. Market Intell Plann 2008; 26: 441-58.

63. Lejoyeux M., Mathieu K., Embouazza H., Huet F., Lequen V. Prevalence of compulsive buying among customers of a Parisian general store. Compr Psychiatry 2007; 48: 42-6.

64. Lau J., Ioannidis J. P., Terrin N., Schmid C. H., Olkin I. Evidence based medicine: the case of the misleading funnel plot. BMJ 2006; 333: 597.

65. Dittmar H. Understanding and diagnosing compulsive buying. In: Coombs R. H., editor. Handbook of Addictive Disorders: A Practical Guide to Diagnosis and Treatment. Hoboken, NJ: Wiley; 2004, pp. 411-50.

66. Rose P., Segrist D. J. Difficulty identifying feelings, distress tolerance and compulsive buying: analyzing the associations to inform therapeutic strategies. Int J Ment Health Addict 2012; 10: 927-35.

67. Kessler R. C., McGonagle K. A., Zhao S., Nelson C. B., Hughes M., Eshleman S. et al. Lifetime and 12-month prevalence of DSM-III-R psychiatric disorders in the United States: results from the national comorbidity survey. Arch Gen Psychiatry 1994; 51: 8-19.

68. Takeuchi D. T., Chung R. C.-Y., Lin K.-M., Shen H., Kurasaki K., Chun C.-A. et al. Lifetime and twelve-month prevalence rates of major depressive episodes and dysthymia among Chinese Americans in Los Angeles. Am J Psychiatry 1998; 155: $1407-14$.

69. García I., Jódar L., Merello P., Santonja F.-J. A discrete mathematical model for addictive buying: predicting the affected population evolution. Math Comput Model 2011; 54: 1634-7.

70. Saad G. Evolutionary consumption. J Consult Psychol 2013; 23: $351-71$.

71. Billieux J, Schimmentic A, Khazaald Y, Mauragea P, Heerena A. Are we overpathologizing everyday life? A tenable blueprint for behavioral addiction research. J Behav Addict 2015; 4: 119-23.

72. Maraz A, Király O, Demetrovics Z. The diagnostic pitfalls of surveys: if you score positive on a test of addiction, you still have a good chance not to be addicted. A response to Billieux et al. 2015. J Behav Addict 2015; 4: 151-54.

\section{Supporting information}

Additional supporting information may be found in the online version of this article at the publisher's web-site:

Funnel plots to account for publication bias. 